\title{
Using Crash Databases to Predict Effectiveness of New Autonomous Vehicle Maneuvers for Lane- Departure Injury Reduction
}

Björn Olofsson and Lars Nielsen

The self-archived postprint version of this journal article is available at Linköping University Institutional Repository (DiVA):

http://urn.kb.se/resolve?urn=urn:nbn:se:liu:diva-172144

N.B.: When citing this work, cite the original publication.

Olofsson, B., Nielsen, L., (2020), Using Crash Databases to Predict Effectiveness of New Autonomous Vehicle Maneuvers for Lane-Departure Injury Reduction, IEEE transactions on intelligent

transportation systems (Print). https://doi.org/10.1109/TITS.2020.2983553

Original publication available at:

https://doi.org/10.1109/TITS.2020.2983553

Copyright: Institute of Electrical and Electronics Engineers

http://www.ieee.org/index.html 


\title{
Using Crash Databases to Predict Effectiveness of New Autonomous Vehicle Maneuvers for Lane-Departure Injury Reduction
}

\author{
Björn Olofsson and Lars Nielsen
}

\begin{abstract}
Autonomous vehicle functions in safety-critical situations show promise in reducing the risk and saving lives in accidents compared to existing safety systems. Consequently, it is from many perspectives advantageous to be able to quantify the potential benefits of new autonomous systems for vehicle maneuvers at-the-limit of tire friction. Here, to estimate the potential in terms of saved lives and reduced degree of injuries in accidents for new, not yet existing systems, a framework has been developed by combining available historic data, in the form of crash databases, and statistical methods with comparative calculations of vehicle behavior using numerical optimization rather than simulation. The framework performs effectively, it gives interesting insights into the relation between more traditional active yaw control and optimal autonomous lanekeeping control, and it clearly demonstrates the potential of saved lives by using autonomous vehicle maneuvers.
\end{abstract}

Index Terms-Risk analysis, active safety, vehicle stability control, extended yaw control, vehicle-braking strategies.

\section{INTRODUCTION}

Autonomous driving has potential to reduce the number of severe vehicle accidents and to save lives, and it is therefore of interest to investigate the potential severity reduction in accidents possible to obtain with new autonomous safety functions and maneuvers in time-critical at-the-limit situations. Knowledge about previous actual accidents is available in large databases (see [1], [2] for examples) that are well structured regarding type of accident, situation parameters, and outcome in terms of degree of injuries and fatalities. Such databases have been used in retrospective studies for quantifying actual performances of different safety systems such as electronic stability control (ESC) [3] and lane-departure warning (LDW) and lane-keeping aid (LKA) [4]. For natural reasons, these databases only contain the outcome of accidents with vehicle control systems that already exist, so a major research question is how such collected and well structured data can be utilized to estimate the potential benefits of a future autonomous safety system. Methods based on simulation and statistical methods have been developed for estimation of potential benefits of vehicle safety systems in, e.g., [5], [6]. Benefit estimations established based on crash databases have been presented for

B. Olofsson and L. Nielsen are with the Div. of Vehicular Systems, Dept. of Electrical Engineering, Linköping University, Sweden. B. Olofsson is also with the Dept. of Automatic Control, Lund University, Sweden.

The authors are members of the ELLIIT Strategic Research Area, supported by the Swedish Government. This research was partially supported by the Wallenberg AI, Autonomous Systems and Software Program (WASP), funded by the Knut and Alice Wallenberg Foundation.

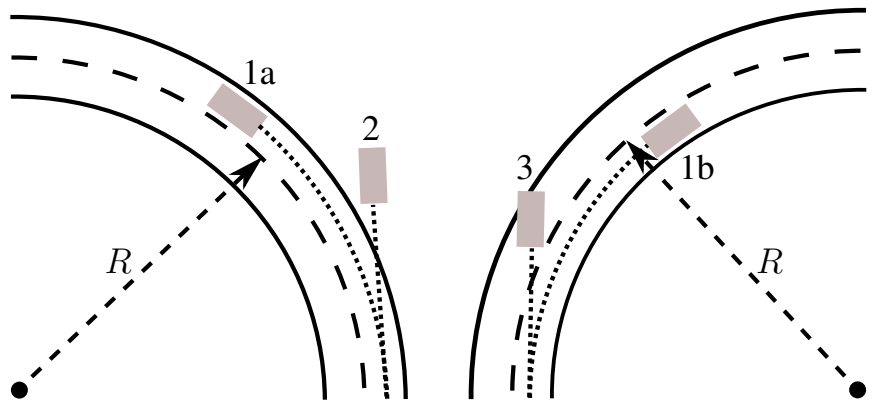

Fig. 1. Possible situations when entering a left-hand or right-hand turn with radius $R$. Cases 2 and 3 can potentially result in accidents, whereas in Cases $1 \mathrm{a}$ and $1 \mathrm{~b}$ the vehicle is able to stay in the desired driving lane.

LDW using statistical comparisons based on simulations of vehicle behavior with and without the safety system active in [7], [8], [9], [10]. Injury risk functions (see, e.g., [11]) were used for prediction of the degree of injury in the simulated accidents with the investigated safety system active.

Regarding the potential of future autonomous vehicles, a first observation when analyzing accidents in a crash database is that if a driver enters a scenario with too high speed it can be physically impossible to avoid lane departure. Thus, for each accident, there is a need to find the limiting behavior to investigate if it would have been possible to stay in lane. If it is not possible to stay in lane even with optimal steering/braking, then the interesting question is how much the speed could be reduced by these maneuvers to mitigate the outcome to milder than fatal or severe injury. Searching for limiting cases, the nature of simulation is that inputs are needed for representing the driver actions, like steering and braking, which may be difficult and time consuming to find for an optimal maneuver, potentially requiring trial and error approaches. Here, optimization immediately gives the limiting case and finds the limit if an accident is possible to resolve or mitigate. This is a fundamental advantage of using optimization, and thus the methodological framework developed in the current paper is based on optimization instead of simulation and it turns out to be computationally sound and effective.

The optimization-based framework for traffic-safety analysis presented here is general but is advantageously presented using an example, and the example chosen is lane-departure situations (see Fig. 1) where active vehicle stabilization is required (possibly on the limit of tire friction). Lane departure is a very critical situation, and this is an area where a 
new line of development is to use more information for the combined steering and braking strategy, specifically to use lane borders and possibly additional situation awareness. The possible situations in the case of lane following and lane departure are depicted in Fig. 1. Note that leaving the lane can mean either to departure from the road (called road-departure accidents) or to enter into the lane of opposing traffic (called opposing-lane accidents). The union of these sets of accidents is called lane-departure accidents.

From a perspective of policies and strategies for future development of autonomy, the outcome of the present study will provide guidance. If it turns out that few severe or fatal accidents could be avoided, then the argument for autonomy would weaken and efforts should be put on other developments. Further, it is of interest to study if an autonomous safety system of lower complexity could avoid few severe or fatal accidents, but a comparably more complex system could avoid significantly more. These questions will be investigated and the outcome turns out to be very clear. A remark, in the context of safety analysis in general, is that the research questions posed in this paper are along the lines of what can be achieved with an autonomous system regarding reduced injuries compared to the outcome of real accidents. Then, there are well established and formal procedures, such as ISO-26262 [12], on how to design a system to achieve this potential. Thus, the method developed here is a valuable complement to existing methods.

\section{Problem Formulation And Contributions}

The main research question posed in this paper is to what extent a new autonomous control principle for vehicle stabilization using combined optimal braking and steering can reduce the risk for severe or fatal injuries in traffic, and what that could mean in terms of reduced degree of injury or saved lives in lane-departure accidents. To this end, comprehensive crash databases [1], [2], extensively used, e.g., by researchers and insurance companies, are available. GIDAS [1] is one such database, described in more detail in Sec. III. Searches by means of filtering are easy to perform such that different types of accidents and their physical characteristics (such as vehicle speed, road curvature, approach angle, and if certain driver-assistance systems were available) can be readily found. For the accident scenarios depicted in Fig. 1, a total of 266 lane-departure accidents have been extracted from GIDAS with the approach described in Sec. III. These accidents are plotted in Fig. 2, there characterized by the initial speed of the vehicle and road curvature. The accidents have been plotted with different colors based on whether an ESC system was installed in the vehicle (though not necessarily if it was active during the particular accident, since that is not known from the database). From the distribution of the accidents with respect to radius and velocity, it is clear that, as expected, there are more accidents with high velocity at low radius and that there are fewer accidents for large radii especially for low velocities.

For the scenarios discussed in the previous paragraph, two possible vehicle-safety systems are compared. One is a version of traditional active yaw control (AYC), see, e.g., [13], and the other is the emerging new autonomous strategy

\section{Road-Departure and Opposing-Lane Accidents}

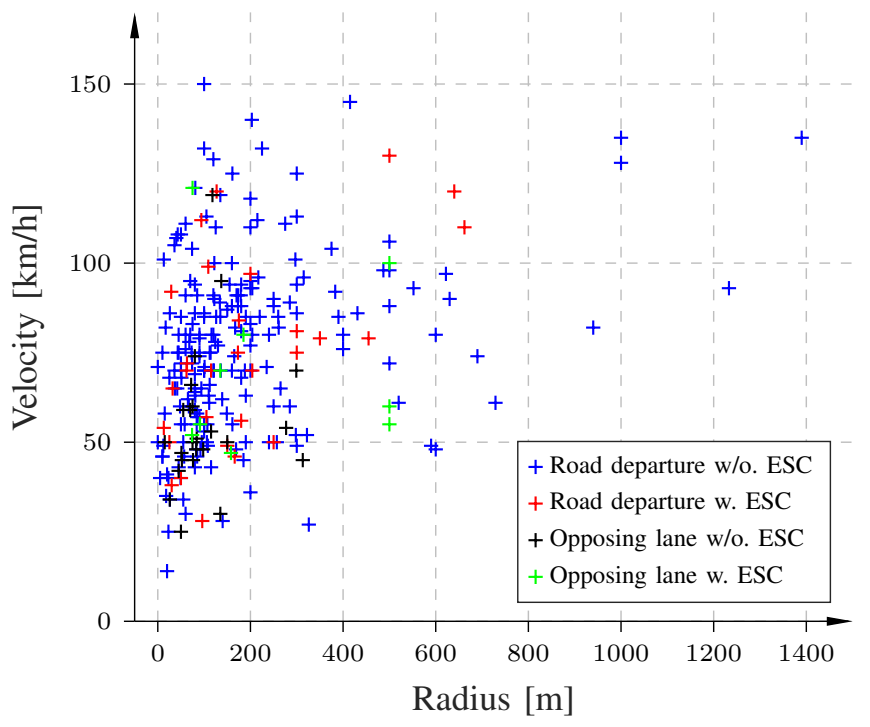

Fig. 2. Illustration of radius and initial velocity for all accidents recorded in the database GIDAS in a turn (with radius less than $1500 \mathrm{~m}$ ) that resulted in departure from the road or into the lane of the opposing traffic and caused a severe injury or fatality. The colors indicate the type of accident and if an ESC system was installed in the vehicle.

employing situation awareness combined with optimal braking and steering, here called optimal lane-keeping control (OLKC) [14] (see also [15] for a related approach to optimal lane keeping), that has been proposed but still does not exist in production vehicles. The control systems are further described in Sec. IV. A key idea in this paper is to compute quantitative characteristics of AYC and O-LKC, such as vehicle state, optimal driver inputs, and braking strategy during the accident scenario, by utilizing recent developments in computation of vehicle behavior at-the-limit of tire friction [16], [17], and using advances in dynamic optimization and associated tools [18]. This basis in vehicle dynamics is important in the analysis of this kind of accidents where the tire forces typically are at their limits. The vehicle modeling and optimization approach employed in this paper is discussed in Sec. V. In Secs. VI-VII, it is described how the so obtained quantitative characteristics of vehicle behavior (from optimization) are incorporated and used in the overall computational framework for benefit estimations. The overall algorithm can be described as follows. In a first step, the detailed information about the vehicle and the particular information about the actual accident is used to determine those accidents that are predicted to be possible to handle with the autonomous system under study (in this respect, the method is general since it can utilize a wide range of detailed information that is available in the database). Subsequently for the subset of accidents that were estimated to be unavoidable in the first step, the algorithm computes in the next step how much the autonomous safety system would decrease the speed before a potential crash and the consequential mitigation of severe and fatal accidents, while taking uncertainty into account. This algorithm creates an effective computational structure and forms a significant contribution in 
the paper, achieved by benefiting from combining extensive data existing in crash databases with modern optimization tools. Finally, the total potential of the safety system in terms of saved lives and injury reductions is summarized in Sec. VIII. Consequently, that section presents a method that is able to estimate the potential benefits of a new control system, already before taking the cost of developing it. The results from application of the method on the lane-departure accidents are also presented. The potential implications of the method and the results from application of it are treated in the conclusions, Sec. IX.

\section{SCEnARIOS AND CRASH DATABASES}

The first step preceding the analysis of possible benefits of a new safety system is to describe the scenarios in terms such that they can be related to accident analysis using accidents available in databases. In this section, the possible cases for the example scenario of lane departure are first described in such a way that they can be extracted from the GIDAS crash database, and then the specific method of applying appropriate filtering criteria is described for the lane-departure example.

\section{A. Scenarios Considered}

The scenario considered in this paper is lane departure, which was mentioned previously and is depicted in Fig. 1. As investigated in [19], it holds that for the accidents in the GIDAS database, the number of lane-departure accidents resulting in collisions with other traffic participants (multi-vehicle scenarios) is slightly higher than collision with other objects (single-vehicle scenarios). However, as shown in Fig. 16 in [19], the injury outcomes from collisions with other objects are more severe than multi-vehicle accidents, with at least the double number of accidents resulting in severe or fatal injuries. Consequently, choosing the most severe accident type, singlevehicle accidents caused by lane departure are considered in the application of the developed method. This scenario of lane departure in right-hand or left-hand turns is often caused by entrance into the curve with a velocity that is higher than what the driver can handle, or even higher than what the vehicle can perform when combining optimal braking and steering under consideration of the available interaction friction between the tires and the road. If right-hand side traffic is assumed and the road is a left-hand turn, then the risk is to leave the road out in the terrain, whereas if it is a right-hand turn, then the risk is to leave the driving lane into the opposing lane. There are two possible outcomes of using an active safety system in a particular driving situation. The first possibility is where the critical situation is handled and the vehicle is able to stay within its current driving lane (situations denoted $1 \mathrm{a}$ and $1 \mathrm{~b}$ in Fig. 1). The other two possible cases are when the vehicle leaves the current driving lane into either the opposing lane (situation denoted 3 in Fig. 1) or when the vehicle leaves the road completely (situation denoted 2 in Fig. 1), possibly colliding. In these two cases, it is of interest to estimate if the risk of severe injury or fatality can be mitigated or even completely be avoided with a certain control principle. The framework developed for the analysis should thus be able
TABLE I

FILTERING CRITERIA USED FOR EXTRACTING THE SOUGHT ROAD-DEPARTURE ACCIDENTS FROM THE GIDAS DATABASE. THE RESULTING ACCIDENTS ARE SHOWN IN FIG. 2, THERE DESCRIBED BY THE RADIUS OF THE TURN AND THE INITIAL VELOCITY.

\begin{tabular}{|l|l|}
\hline Filter & No. \\
\hline Participants & 50432 \\
\hline Thereof M1 vehicles & 32853 \\
\hline Thereof single vehicle accidents & 5428 \\
\hline Thereof loss of control accidents & 2689 \\
\hline Excluding driver incapacity & 2465 \\
\hline Thereof road departure & 1594 \\
\hline Thereof in curve & 800 \\
\hline Thereof severe or fatal injuries & 305 \\
\hline Initial velocity and radius known & 233 \\
\hline Thereof with ESC & 29 \\
\hline Thereof without ESC & 202 \\
\hline Thereof with unknown ESC & 2 \\
\hline
\end{tabular}

to handle both the case when the accident is predicted to be possible to be completely avoided, and the case when only a reduction of velocity is estimated to be possible, but this velocity reduction is such that severe or fatal injuries potentially could be avoided.

\section{B. Crash Databases}

Crash databases containing records from previous real vehicle accidents comprise a rich material, where a significant amount of relevant data about the scenario and the conditions before and during the crash are stored, see, e.g., [19]. Based on the information from such databases, a spectrum of different analyses can be performed. Examples are estimation of functions predicting the probability for risk of injury using different predictor quantities as input to the model (see, e.g., [11]). One example of a database with extensive information is the German In-Depth Accident Study (GIDAS) [1], which is the data source employed for the analysis presented in this paper. Further analysis of a subset of the accidents in GIDAS is available in the Pre Crash Matrix (PCM) database [6], where the trajectories of the vehicle pre-accident have been reconstructed for a subset of the complete database.

For the scenario in Fig. 1, the accidents with severe or fatal injuries in GIDAS have been filtered out based on a number of criteria. The filtering process is a sequence of extraction criteria, and the outcome of the filtering, resulting in the set of road-departure accidents, is shown in Table I. In the table, it is seen that there are 233 accidents in that group, where the speed and road curvature are known. The case of opposing lane is done analogously and that process results in 33 accidents. These are the in total 266 lane-departure accidents that are plotted in Fig. 2.

\section{Active Safety Systems to Compare}

Safety systems in vehicles relying on preview information and knowledge about the ego vehicle have had an enormous development during the last decades, and autonomy and autonomous functions hold promise of yet another level of safety. In connection with this aspect, it should be noted that introduction of new sensor systems potentially could bring new 
safety challenges, where well-known examples are the risk of misclassification of obstacles. However, for sensor systems relevant to lane departure the safety risk is considered low, and the reason is already available sensor systems in production for LDW and LKA systems, so the whole area is consolidating. Further, it is likely that information in maps of the road network can be used to assist the onboard sensing system, e.g., to provide further robustness to online curvature estimation.

For lane departure, two safety strategies called O-LKC and AYC are evaluated in the analysis. The systems are selected such that one, O-LKC, represents an advanced system based on optimal steering in combination with optimal braking strategies, whereas the other represents a straightforward extension of AYC (by braking only) with a steering law simply based on road curvature. The systems will be investigated and quantified with respect to what potential benefit the suggested control principle could provide in terms of decrease in severe injuries or fatalities in the case of lane-departure situations.

\section{A. Active Yaw Control (AYC)}

Systems for AYC use interpretation of vehicle state and driver inputs (such as speed and steering-wheel input) to deploy a braking strategy to obtain corrective yaw moment on the vehicle, see, e.g., [13]. Consequently, in conventional AYC systems, the driver steering input is used in combination with an automatic braking strategy. Thus, there is a need for a steering model, and here the traditional wisdom is used that the driver should be steering in the direction of the road.

Simple Steering Model for AYC: The steering in the AYC system is thus modeled to be in the direction of the road. For a turn with constant radius $R$, this implies a steering angle

$$
\delta_{s}=L / R
$$

where $L$ is the length of the vehicle wheelbase. The steeringangle input according to (1) is low-pass filtered with a firstorder filter with a time constant of $T_{s}=0.1 \mathrm{~s}$ according to

$$
\delta=\frac{1}{s T_{s}+1} \delta_{\mathrm{s}},
$$

to obtain the actual steering angle $\delta$ of the front wheels.

Dual Interpretation of Steering Model for AYC: When a vehicle maneuver is performed by a human driver, the steering control law in (1) describes what an alert and correctly acting driver would do. Consequently, the model in the previous paragraph represents AYC with such a driver. On the other hand, if the steering control law (1) is implemented as an additional part of an AYC system relying on braking, the complete system then becomes an autonomous safety system that controls both braking and steering, with an intuitive steering strategy, though not optimal as the steering pattern in O-LKC that will be detailed in the next subsection. This modeling of AYC will naturally give inferior performance compared to a system with also optimal steering.

\section{B. Optimal Lane-Keeping Control (O-LKC)}

A new line of development for safety systems is to use more information, e.g., lane borders and road curvature, which now is available in modern cars as a result of new sensor technology and improved computing power. This information is then used to devise the steering and braking strategy. Such formulations for optimization-based lane-keeping control are found in [14], [17], where lane borders were used as constraints in an optimization of a time-critical turn maneuver. In contrast to the vehicle equipped with $\mathrm{AYC}$, the vehicle with O-LKC is allowed to both brake independently on all wheels and optimally coordinate the steering. Thus, the vehicle control system is free to select the best possible combination of braking and steering inputs. Moreover, the vehicle is also in this case assumed to be operated completely autonomously in the case when the vehicle enters a critical situation and the driver needs assistance for keeping the car on the road. This system is referred to as optimal lane-keeping control (O-LKC).

\section{MODELING AND OPTIMIZATION}

The driving behaviors for the two different control principles are computed using numerical solutions of optimal control problems. The road-geometry parameters and the initial conditions are extracted as far as possible from the crash database, and are used when the optimization problem is solved for each of the specific accidents. In this section, the modeling of the vehicle dynamics and the subsequent optimal motioncontrol problems for AYC and O-LKC are treated. The same model is used for both vehicles with the respective control system, while the differences in behavior between the two control principles are established using the formulation of the objective function in the optimization problem.

\section{A. Modeling of Vehicle Dynamics}

A dynamic double-track (DT) chassis model according to [16] is employed for the computations. The inputs $u$ to the model are the steering angle $\delta$ of the front wheels and the torques $T_{u}$ on the respective wheel. The model comprises both longitudinal and lateral load transfer, which is modeled using spring-mass-damper dynamics characterized by a spring constant and a damping coefficient. The tire-force modeling is essential for an accurate description of the maneuver, since the tire-friction utilization is typically at its limit in this kind of maneuver. Based on the methodology and insights presented in [16], the Pacejka's Magic Formula model [20] is used for describing the tire forces under pure longitudinal tire slip $\kappa$ or pure lateral tire-slip $\alpha$. For combined longitudinal and lateral slip, weighting functions [20] are used. Moreover, wheel dynamics is included in the model, i.e., the wheel torques are used as inputs for the vehicle dynamics. The vehicle chassis and tire models are formulated in the declarative modeling language Modelica [21] as a differential-algebraic equation (DAE) with the functions $G$ and $h$ as

$$
\begin{aligned}
G(\dot{x}, x, z, u) & =0, \\
h(x, z, u) & =0,
\end{aligned}
$$

where $x$ are the states, $u$ the inputs, and $z$ the algebraic variables of the model. The reader is referred to the model called DT WF in [16] for the complete model equations and details regarding the vehicle modeling and the specific model parameters used. 


\section{B. Optimal Control Problem}

The computational approach for obtaining optimal vehicle behaviors in the respective accident scenario is based on the optimization platform presented in [16]. That platform was also employed in [14] to quantify the performance increase in technical terms possible to achieve with optimal lane-keeping control compared to conventional AYC. In order to compare the two different vehicle-safety systems in different scenarios, a series of optimization problems are solved, both for computing the maximum manageable velocity curves (see Sec. VI-B) and for predicting departure velocities (see Sec. VII). The method for solving the problems is described in detail in [17], and is therefore only shortly recapitulated here. In [17], it was found that the driving behaviors corresponding to the control principles of AYC and O-LKC can be obtained by optimization of the exit velocity $v_{f}$ and the initial velocity $v_{0}$ of the manuever, respectively. Consequently, these variables are used as optimization objectives here. Further, certain constraints are enforced in the optimization. More specifically, the wheel torques $T_{u}$ are constrained because of braking limitations and the steering angle $\delta$ and its corresponding change rate are constrained since the wheels cannot instantaneously change their heading direction. The optimal control problem to be solved over $\left[0, t_{f}\right]$ is stated as (see [17] for further details):

$$
\begin{aligned}
\operatorname{minimize} & -v_{0} \text { or }-v_{f} \\
\text { subject to } & T_{u, i, \min } \leq T_{u, i} \leq 0, i \in\{1,2,3,4\}, \\
& |\delta| \leq \delta_{\max },|\dot{\delta}| \leq \dot{\delta}_{\max } \\
& f\left(X_{p}, Y_{p}\right) \leq 0, g\left(x\left(t_{f}\right)\right) \leq 0 \\
& F_{c} x(0)=\tilde{x}_{0}, G_{c} x\left(t_{f}\right)=\tilde{x}_{f} \\
& G(\dot{x}, x, z, u)=0, h(x, z, u)=0
\end{aligned}
$$

where $f$ defines the path constraints on the global vehicle position $\left(X_{p}, Y_{p}\right), g$ defines the terminal inequality constraint, and $F_{c}, G_{c}$ determine the initial and final constraints. The two optimal control problems to solve for AYC and O-LKC are thus obtained by minimizing $-v_{f}$ or $-v_{0}$, respectively.

\section{Solution of Optimal Control Problem}

For solving the optimization problem (5)-(10) numerically, the optimization software JModelica.org [18] is used. This platform provides algorithms for numerical optimization based on dynamic systems implemented using the Modelica modeling language as a DAE system on the format (3)-(4). For a fair comparison of AYC and O-LKC, all vehicle and tire parameters are equal for both cases. This also holds for all initial and operational conditions. Since O-LKC has more information about the driving situation available, it can potentially be aware of a critical situation sooner than AYC that relies on detection of a critical situation based on sensor data [22], so called triggering events. This particular potential advantage for O-LKC is not exploited in the comparison.

\section{Framework for Prediction of Potentially AVOIDED SEVERE ACCIDENTS}

The approach used to predict the potential benefits of a new safety system has two components, both building on the optimization framework in Sec. V. First, there is a need to estimate the capability of the vehicle to stay in lane for each of the accidents in the crash database, and if it is not possible to stay in lane to estimate a crash velocity in an impact situation. For the latter group of accidents, information from the crash database is used to estimate the mitigation of risk, i.e., the potentially reduced severity of injuries and reduced number of fatalities in the accidents.

To analyze the potential benefits of a specific active-safety system, the following phases are considered in the computational framework developed:

- Estimate the number of situations where the vehicle equipped with a certain autonomous safety system can stay in lane, given the available tire friction. This computational procedure is described in Sec. VI-B.

- The second estimation to be performed is for the subset of situations where the vehicle leaves the road into the terrain or into the opposing driving lane, despite that the active-safety system is in operation. It is clear that a reduction of velocity before impact should decrease the probability of a severe injury or even fatality in an accident. It is therefore in these cases of interest to study the reduction of vehicle velocity that is obtained with the specific safety system active, before the crash occurs. Consequently, there is a need to estimate the departure velocity $v_{\mathrm{D}}$ in these cases. The computational approach for this purpose is described in Sec. VII-C.

- With the predicted departure velocities, computed for the accidents where the car is not able to stay on the road, the obtained data are used in a statistical method to compute a distribution of the number of severe accidents that are mitigated with the considered safety system, where injury risk functions are used to predict the outcome of an accident, given the predicted impact velocity. The computational approach for this is described in Sec. VII-D.

- Finally, the computational framework gives an estimate of how many accidents with severe of fatal injuries in the selected set of accidents the investigated control principle potentially could avoid, either by complete avoidance or mitigation. The complete algorithm is given in Sec. VIII.

\section{A. Terminology and Definitions}

To formulate the method, certain terminology is introduced and a set of definitions is established. Let $\mathcal{A}$ denote the set of all accidents $a_{i}, i=1, \ldots, N$, in a database containing

$$
N=\operatorname{card}(\mathcal{A}),
$$

entries fulfilling the desired filtering criteria (concerning, e.g., type of accident and driver conditions such as in Table I). For each accident $i$, the initial velocity of the vehicle is $v_{0}^{i}$. It is of interest to study the subset of the complete set $\mathcal{A}$ that can be managed using a new control principle to be investigated. To this purpose, the maximum initial velocity $v_{\mathrm{M}}$ that could be handled with a certain control system is defined. This quantity, called the manageable velocity, is assumed to be a function of the following critical parameters:

$$
v_{\mathrm{M}}=v_{\mathrm{M}}\left(R ; \gamma_{\mu}, d\right)
$$


where $R$ is the radius of the turn, $\gamma_{\mu}$ is the scaling constant of the friction coefficients, and $d$ is the lane width (corresponding to the maximum maneuvering limit for the vehicle center-ofgeometry, see further details in [17]). The subset of $\mathcal{A}$ with accidents that are predicted to be possible to handle with the evaluated control strategy is called the manageable (M) accident set and is defined as

$$
\mathcal{A}_{\mathrm{M}}=\left\{a_{i} \mid v_{0}^{i} \leq v_{\mathrm{M}}\left(R ; \gamma_{\mu}, d\right)\right\} .
$$

Further, introduce the notation

$$
N_{\mathrm{M}}=\operatorname{card}\left(\mathcal{A}_{\mathrm{M}}\right) \text {. }
$$

The accidents not contained in $\mathcal{A}_{\mathrm{M}}$, i.e., the accidents that are predicted not to be possible to handle with the evaluated control strategy, is consequently contained in the complement

$$
\mathcal{A}_{\mathrm{nM}}=\mathcal{A} \backslash \mathcal{A}_{\mathrm{M}}
$$

being the non-manageable (nM) accidents. For the accidents in this set with size

$$
N_{\mathrm{nM}}=\operatorname{card}\left(\mathcal{A}_{\mathrm{nM}}\right),
$$

it is of interest to estimate what the velocity in the impact situation would be, if the new control principle would have been active and then probably has reduced the velocity by braking. Hence, this is the predicted departure velocity $v_{\mathrm{D}}$.

\section{B. Maximum Manageable Velocity Curves}

The maximum manageable velocity curve for a certain control principle is naturally dependent on the friction between the tire and the road. The nominal case, corresponding to driving on dry asphalt, is obtained when $\gamma_{\mu}=1$. The maximum manageable velocity curve $v_{\mathrm{M}}$ was computed for $\mathrm{O}-\mathrm{LKC}$ as function of the radius of the turn using the method described in Sec. V, i.e., by solving the optimization problem (5)-(10) for a given set of radius values $R$. Thereby, dry asphalt conditions were assumed for the road. In Fig. 3, the maximum manageable velocity curve, computed for a number of radii in the interval $R \in[0,300] \mathrm{m}$, and the accidents extracted from the GIDAS database are plotted as functions of the turn radius $R$ in the case that the lane width is $d=1 \mathrm{~m}$. Accidents below the curve, i.e., in the set $\mathcal{A}_{\mathrm{M}}$, thus have the potential to be handled by O-LKC under the given conditions.

The maximum manageable velocity curves $v_{\mathrm{M}}$ were also computed for both O-LKC and AYC for different values of the scaling constant for the friction coefficients. The results for the values $\gamma_{\mu} \in\{0.75,1\}$ and the lane width $d=1 \mathrm{~m}$ are collected in Fig. 4.

\section{Results for $A Y C$ and $O-L K C$}

The accidents employed in the analysis are shown in Fig. 5, where the maximum manageable velocity curves $v_{\mathrm{M}}$ are shown for AYC and O-LKC in the case when all friction coefficients are scaled with $\gamma_{\mu}=0.75$. These values of the tire-friction coefficients correspond to a slightly slippery road surface, such as after rain on an asphalt road. This is the value of $\gamma_{\mu}$ used for the subsequent analysis of the respective vehicle control
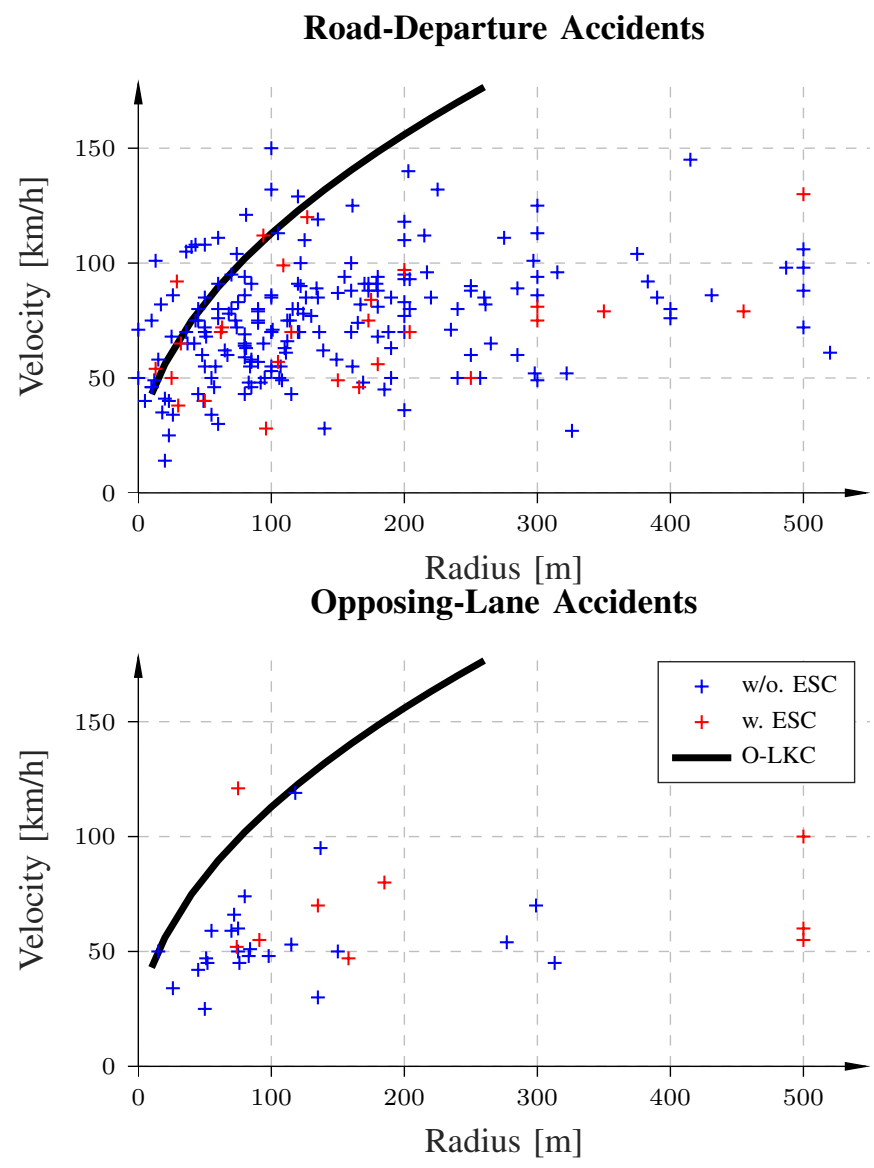

Fig. 3. Maximum manageable velocity $v_{\mathrm{M}}$ as function of the turn radius $R$ for O-LKC in the case that the lane width $d=1 \mathrm{~m}$, plotted together with the road-departure and opposing-lane accidents from GIDAS in the interval $R \in[0,550] \mathrm{m}$ for $\gamma_{\mu}=1$.

system. For the road-departure accidents, there are $N_{\mathrm{nM}}=43$ accidents above the maximum manageable velocity curve $v_{\mathrm{M}}$ for O-LKC and $N_{\mathrm{M}}=177$ accidents are below the maximum manageable velocity curve for AYC. A total number of 13 accidents are in the grey shaded area between the maximum manageable velocity curves for O-LKC and AYC. For the opposing-lane accidents, there are $N_{\mathrm{nM}}=3$ accidents above the maximum manageable velocity curve $v_{\mathrm{M}}$ for O-LKC and $N_{\mathrm{M}}=30$ accidents are below the maximum manageable velocity curve for AYC. No accidents are in the grey shaded area between the maximum manageable velocity curves for O-LKC and AYC.

\section{PREDiction of Departure Velocity AND Mitigation Estimation}

In this section, the computational procedure for prediction of the departure velocity and the potential injury reduction possible to obtain is described. First, maximum manageable velocity curves $v_{\mathrm{M}}$ (see Sec. VI-B) for the considered safety system are used for determining if an accident belongs to $\mathcal{A}_{\mathrm{M}}$ or $\mathcal{A}_{\mathrm{nM}}$. For the accidents in $\mathcal{A}_{\mathrm{nM}}$, a prediction of what the departure velocity $v_{\mathrm{D}}$ would have been, if the new safety system were active, is computed for each individual accident. Based on the departure velocity predicted using 


\section{Road-Departure Accidents}

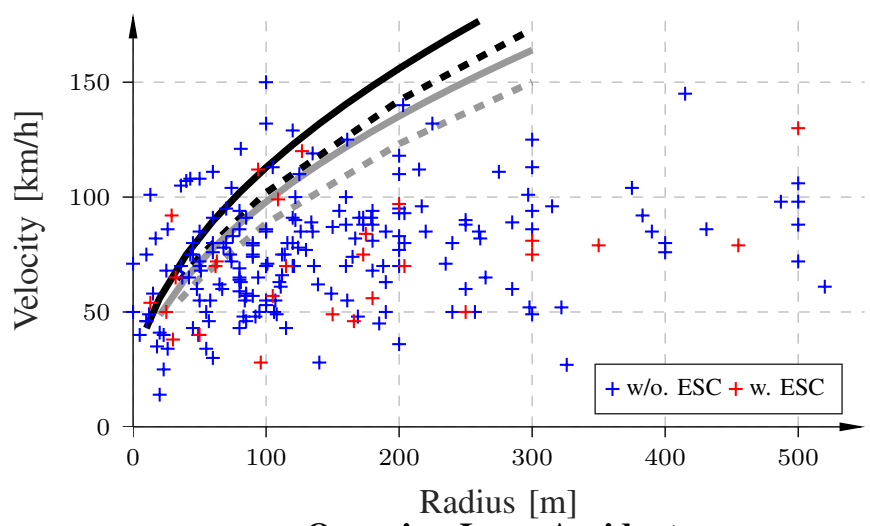

Opposing-Lane Accidents

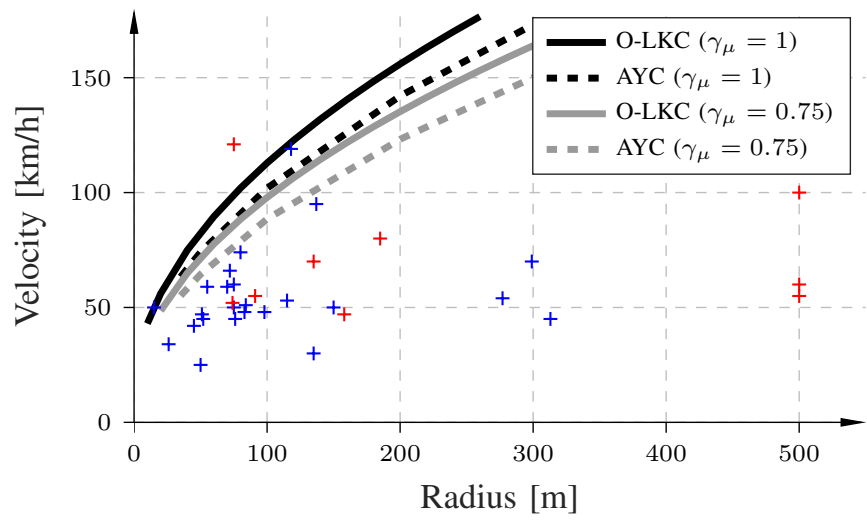

Fig. 4. Maximum manageable velocity $v_{M}$ as function of the turn radius $R$ for different values of the friction coefficients in the tire-force model (described by the scaling factor $\gamma_{\mu}$, implying that all tire-friction coefficients are downscaled equally) and the lane width $d=1 \mathrm{~m}$, using the control principles of AYC and O-LKC, respectively.

the optimization framework in Sec. V, injury risk functions are subsequently used in a statistical method to predict the outcome of the accident in terms of severity of injuries.

\section{A. Manageable Accidents}

Initially, all accidents $a_{i}$ in the set $\mathcal{A}$ are iterated over. If the initial velocity $v_{0}^{i}$ for accident $a_{i}$ is lower than the maximum manageable velocity according to $v_{\mathrm{M}}$ for the considered safety system and the turn radius for the accident, then the accident belongs to the set $\mathcal{A}_{\mathrm{M}}$. If not, the accident is in the set $\mathcal{A}_{\mathrm{nM}}$.

\section{B. Injury Risk Functions}

In Fig. 2, it can be noted that there is a significant spread in velocities and road curvatures, and that severe or fatal injuries can occur also at relatively low speeds. The significance of velocity is different for different types of accident analysis depending on scenario, and in some cases it is an open research question, e.g., in dense freeway traffic [23], [24]. However, when it comes to lane departure, velocity is critical as a significant indicator of the crash energy and thus of injury risk. A well-founded statistical approach to model risk of injury is to use injury risk functions [11] (also known as injury risk
Road-Departure Accidents
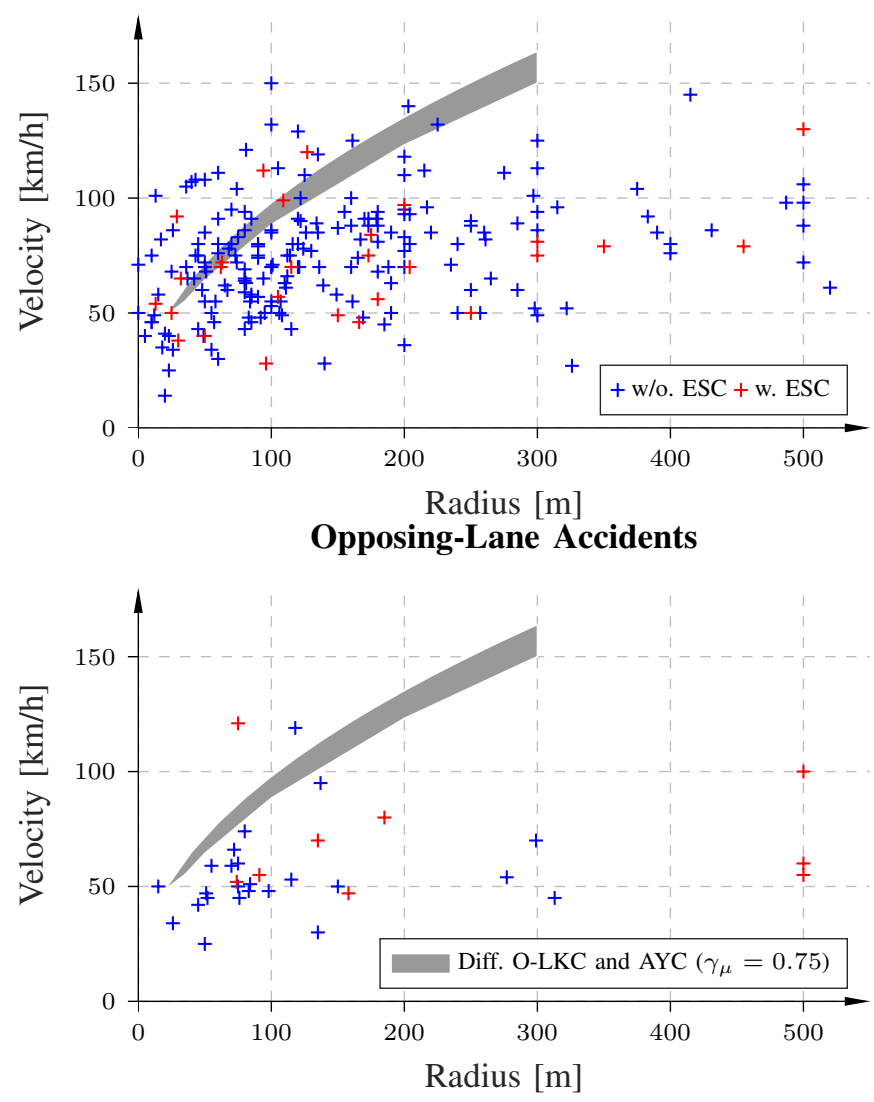

Fig. 5. Maximum manageable velocity $v_{\mathrm{M}}$ as function of the turn radius for AYC and O-LKC for the case when $\gamma_{\mu}=0.75$ and the lane width $d=1 \mathrm{~m}$. The grey shaded areas indicate the velocity interval where AYC and O-LKC differ in terms of what maximum initial velocity they can manage for a certain turn radius.

curves), i.e., functions that predict the probability $p_{r}(\xi)$ of a certain degree of injury in an accident, given input variables $\xi$, as conceptually described by

$$
p_{r}(\xi)=\mathcal{P}(\text { fatal or severe injury } \mid \text { predictors } \xi) .
$$

Regarding the choice of predictors $\xi$, it is noted that there is a high correlation between the kinetic energy involved in the crash and the resulting degrees of human injuries, where change of velocity $\Delta v$ is a main indicator for severe or fatal accidents [11], [19], and thus $\Delta v$ is the natural choice as predictor in the injury risk function. To find such an injury risk function based on $\Delta v$, the accidents from GIDAS could in principle be used. However, injury risk functions based on a much larger data set proposed in [11] are used in the analysis in this paper. They are estimated based on information in data extracted from an extensive database with broad classes of accidents. That database is maintained by the Swedish insurance company Folksam and contains analysis of accidents in the Swedish Traffic Accident Data Acquisition (STRADA) [2] database, which comprises information about previous car accidents in Sweden. Thus, the following injury risk function $p_{r}$ from [11] is used

$$
p_{r}(\Delta v)=\frac{1}{1+e^{-\alpha-\beta \Delta v}},
$$


Injury Risk Function with $\Delta v$ as Predictor

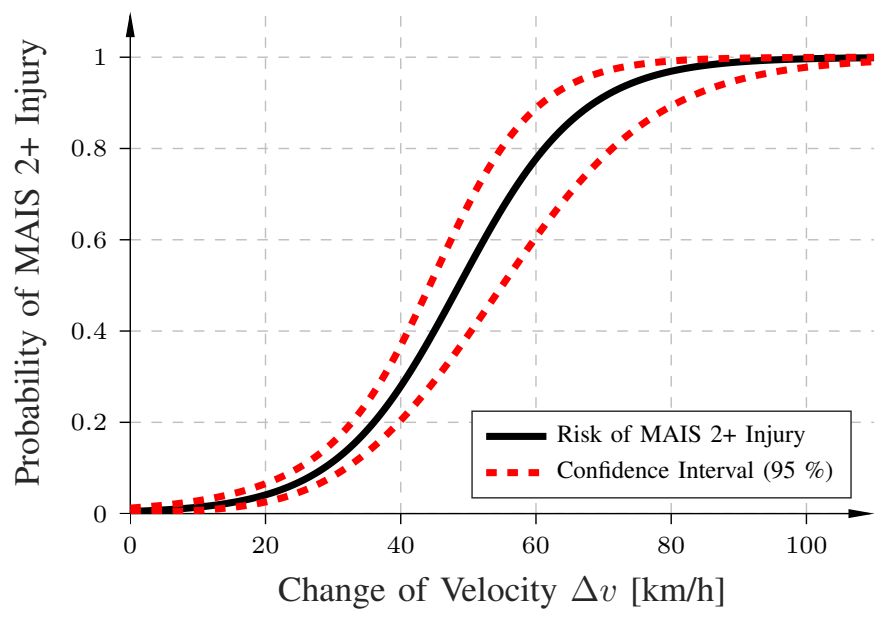

Fig. 6. Injury risk function from [11] for computation of the probability of an MAIS $2+$ injury in an accident, given the change of velocity $\Delta v$ in the crash. The confidence interval has been computed based on the covariance matrix for the regression parameters $\alpha$ and $\beta$ stated in [11].

where $\alpha$ and $\beta$ are the parameters of the function (which are inferred from data using logistic regression [25]). Such an injury risk function with parameters from [11], estimating the risk of an MAIS 2+ injury (corresponding to that the maximum injury was 2 or higher on the abbreviated injury scale (AIS) [26]) in an accident is shown in Fig. 6. The numbers for the covariance matrix for the parameters $\alpha$ and $\beta$ stated in [11] were used for computing the confidence interval and later when sampling the parameters of the function. In the following, it is assumed that the departure velocity $v_{\mathrm{D}}$ equals the total change of velocity $\Delta v$, i.e., $\Delta v=v_{\mathrm{D}}$, when using the injury risk functions, meaning that a complete standstill of the vehicle is assumed after the impact.

\section{Prediction of Departure Velocity}

In this subsection, the method for predicting the departure velocity $v_{\mathrm{D}}$ for the accidents in the set $\mathcal{A}_{\mathrm{nM}}$ is described (cf. Fig. 7). The computations are based on solving the optimization problem (5)-(10) in Sec. V for each of the accidents, thus taking the full dynamic model and the specific road geometry for each accident into account.

1) For accident $a_{i} \in \mathcal{A}_{\mathrm{nM}}$, extract the initial velocity $v_{0}^{i}$ and the curve radius $R_{\mathrm{a}}^{i}$ from the database. If known values of the friction coefficient $\mu=\mu_{0}$ and the lane width $d=d_{0}$ of the road exist, use the actual values. Otherwise, estimates of these values are used.

2) Find the minimum lane width $d$ such that the maximum manageable velocity for the considered safety system is $v_{0}^{i}$ (i.e., the actual initial velocity from the accident database). This computation is performed using optimization, either by fixing the initial velocity to the actual $v_{0}^{i}$ and solving an optimization problem where $d$ is a free variable and the objective is to minimize $d$, or by successively increasing $d$ and then solving for the maximum manageable initial velocity for the sequence

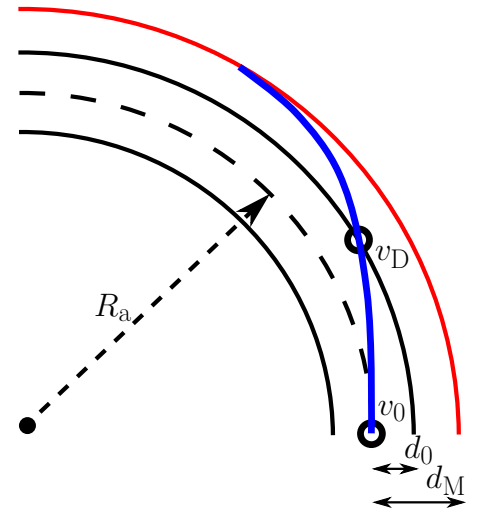

Fig. 7. Schematic illustration of the computation of the departure velocity $v_{\mathrm{D}}$ based on solving a series of optimization problems (see (5)-(10) in Sec. V). The lane width $d_{0}$ (road with black curves) is successively increased (road with red curves) until a solution is found where the computed maximum manageable velocity $v_{\mathrm{M}}$ of the maneuver is sufficiently close to $v_{0}$ for the current accident. The departure velocity is then estimated to be the velocity where the vehicle path (blue curve) intersects with the actual road with lane width $d_{0}$.

of fixed lane widths. Here, the latter approach is used as follows. Successively increase the lane width $d$ of the road according to the iteration

$$
d_{k+1}=d_{k}+d_{\varepsilon}
$$

starting with the initial value $d_{0}$. For each step with increased lane width $d_{\varepsilon}$, find the value of the maximum manageable velocity $v_{\mathrm{M}}\left(R_{\mathrm{a}}^{i} ; \mu_{0}, d_{k}\right)$ according to the optimization procedure (5)-(10) in Sec. V. The iterations are terminated when a solution is found in the optimization such that the maximum velocity $v_{\mathrm{M}}$ is sufficiently close to $v_{0}^{i}$. Denote the corresponding lane width $d_{\mathrm{M}}^{i}$. In the case that the turn radius $R_{\mathrm{a}}^{i}$ is very low and the initial accident velocity $v_{0}^{i}$ is very high, or that no solution to the optimization problem fulfilling that $v_{\mathrm{M}}$ is sufficiently close to $v_{0}^{i}$ for any $d_{k}$ is found, the departure velocity is set to the initial accident velocity, $v_{\mathrm{D}}^{i}=v_{0}^{i}$, and the procedure is continued with the next accident in Step 1.

3) Determine the departure velocity $v_{\mathrm{D}}^{i}$ of the solution of the optimization obtained for solving the problem (5)(10) in Sec. V with $R=R_{\mathrm{a}}^{i}$ and $d=d_{\mathrm{M}}^{i}$ at the point where the outer boundary of the road with lane width $d=d_{0}$ intersects with the computed path. This part of the computational procedure is schematically illustrated in Fig. 7. Return to Step 1 and continue with the next accident.

4) The preceding Steps 1-3 can then be repeated for the complete set of accidents for a set of parameters, where the friction-scaling constant $\gamma_{\mu}$ and the lane width $d_{0}$ are varied, to investigate influence of such variations.

The outcome from this computational algorithm is then predictions of the departure velocity $v_{\mathrm{D}}^{i}$ for each accident $a_{i} \in \mathcal{A}_{\mathrm{nM}}$, i.e., a prediction of what the departure velocity would have been with the considered safety system active. 


\section{Estimation of Mitigation}

In this section, the computational method used for estimating the predicted possible mitigations of the injuries resulting from the accidents in the non-manageable set $\mathcal{A}_{\mathrm{nM}}$ is defined. Denote the number of accidents in $\mathcal{A}_{\mathrm{nM}}$ that are predicted not to result in severe or fatal injuries with application of the considered safety system (as a result of the obtained reduction of velocity) by $N_{\mathrm{B}}$ and the distribution of this variable by $\mathcal{P}_{N_{\mathrm{B}}}$. The method for estimating this number and the corresponding distribution is based on the injury risk functions described in Sec. VII-B. A Monte Carlo simulation approach is used to compute the distribution $\mathcal{P}_{N_{\mathrm{B}}}$ for the considered safety system, under the inherent uncertainty in the injury risk functions. The Steps 1-6 in the following computational procedure are repeated for $k=1, \ldots, K$, where $K$ is a large number, in order to compute the approximate distribution $\mathcal{P}_{N_{\mathrm{B}}}$. The approach relies on the optimization method for prediction of the departure velocity $v_{\mathrm{D}}$ defined in Sec. VII-C.

\section{Estimation of Mitigation Effect- $N_{\mathrm{B}}$ and $\mathcal{P}_{N_{\mathrm{B}}}$}

1) To capture the inherent uncertainty in the injury risk functions, the parameters $\alpha$ and $\beta$ in (18) are drawn according to the probability density function for a Normal distribution $\mathcal{N}(\mu, \Sigma)$, where the mean $\mu$ and covariance matrix $\Sigma$ are obtained from the logisticregression estimation procedure for $\alpha$ and $\beta$ of the particular injury risk function employed, here from [11].

2) Iterate over the accidents $a_{i} \in \mathcal{A}_{\mathrm{nM}}$ for all $i=$ $1, \ldots, N_{\mathrm{nM}}$.

3) Compute $p_{r}(\Delta v)$ for accident $a_{i}$ using the injury risk function with parameters according to the first step, but with the velocity $v_{0}^{i}$ from the accident database for that particular accident $a_{i}$ replaced with the predicted departure velocity $v_{\mathrm{D}}^{i}$.

4) Draw a number from the uniform distribution $\mathcal{U}(0,1)$ to predict if a severe or fatal accident would happen with the safety system under consideration active in the accident (i.e., if the sampled number is less than or greater than the probability computed by the risk function in the previous step).

5) Store the total number $N_{\mathrm{B}}^{k}$ of accidents predicted not to result in severe or fatal injuries for the safety system, i.e., those accidents contained in the set $\mathcal{A}_{\mathrm{nM}}$ that could be mitigated by the decrease of velocity as a result of the braking obtained by the safety system considered.

6) Return to Step 2 and continue with the next accident.

The result of this computational procedure is an estimate of the expected mitigation value $N_{\mathrm{B}}$, and its estimated distribution $\mathcal{P}_{N_{\mathrm{B}}}$, based on all non-manageable accidents in $\mathcal{A}_{\mathrm{nM}}$.

\section{E. Results for AYC and O-LKC in Road-Departure Accidents}

The road-departure accidents from GIDAS are used in the subsequent analysis presented in this and the next sections. To establish an approximate distribution for $N_{\mathrm{B}}$, the computational procedure in Sec. VII-D was repeated $K=20000$ times. The values of the computed $N_{\mathrm{B}}^{k}$ for $k=1, \ldots, K$, can

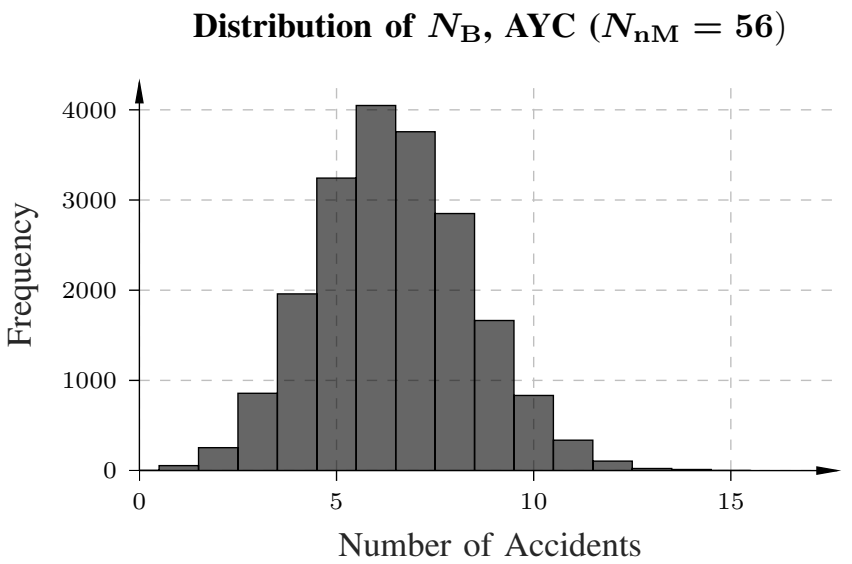

Fig. 8. Histogram showing an approximation of the distribution $\mathcal{P}_{N_{\mathrm{B}}}$ for the number $N_{\mathrm{B}}$ in the case of AYC for road-departure accidents, out of the $N_{\mathrm{nM}}=56$ accidents that are above the maximum manageable velocity curve The histogram was computed for $K=20000$ Monte Carlo iterations.

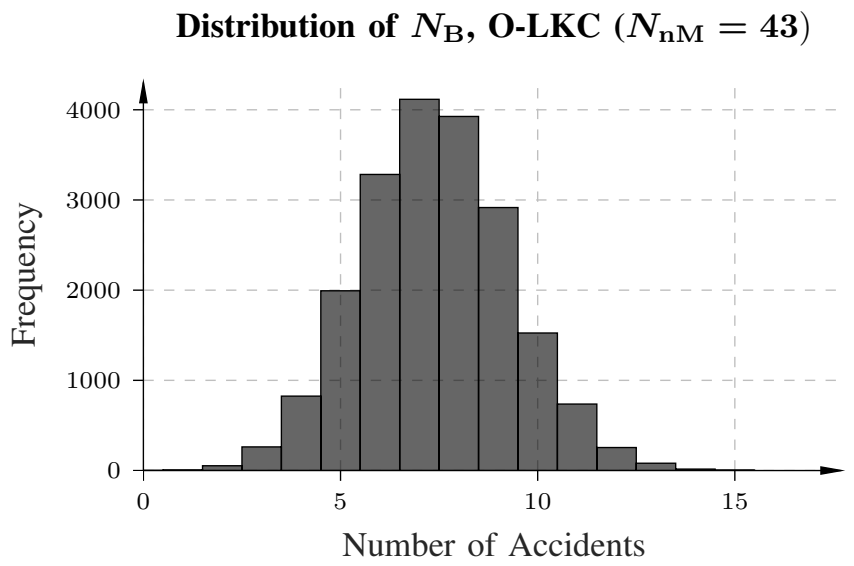

Fig. 9. Histogram showing an approximation of the distribution $\mathcal{P}_{N_{\mathrm{B}}}$ for the number $N_{\mathrm{B}}$ in the case of O-LKC for road-departure accidents, out of the $N_{\mathrm{nM}}=43$ accidents that are above the maximum manageable velocity curve. The histogram was computed for $K=20000$ Monte Carlo iterations.

be visualized in a histogram to give an approximation of the underlying distribution $\mathcal{P}_{N_{\mathrm{B}}}$.

The histogram for the computed $N_{\mathrm{B}}^{k}$ for $k=1, \ldots, K$, in the case of road-departure accidents and AYC is shown in Fig. 8. From the data shown in the histogram, the arithmetic mean of the distribution is computed to be $N_{\mathrm{B}}=6$ accidents. Thus, out of the total $N_{\mathrm{nM}}=56$ accidents considered, six are predicted to be possible to mitigate such that they do not result in severe or fatal injuries (i.e., $11 \%$ of the $N_{\mathrm{nM}}$ accidents).

A corresponding histogram for the resulting $N_{\mathrm{B}}^{k}$ in the case of road-departure accidents and O-LKC is shown in Fig. 9. From the data in that histogram, the arithmetic mean of the distribution is computed to be $N_{\mathrm{B}}=7$ accidents. Thus, out of the total $N_{\mathrm{nM}}=43$ accidents considered, seven are predicted to be possible to mitigate such that they do not result in severe or fatal injuries (i.e., $16 \%$ of the $N_{\mathrm{nM}}$ accidents).

\section{TOTAL INJURY REDUCTIONS}

In this section, the complete algorithm for computing the potential of the safety system in terms of avoided accidents and 


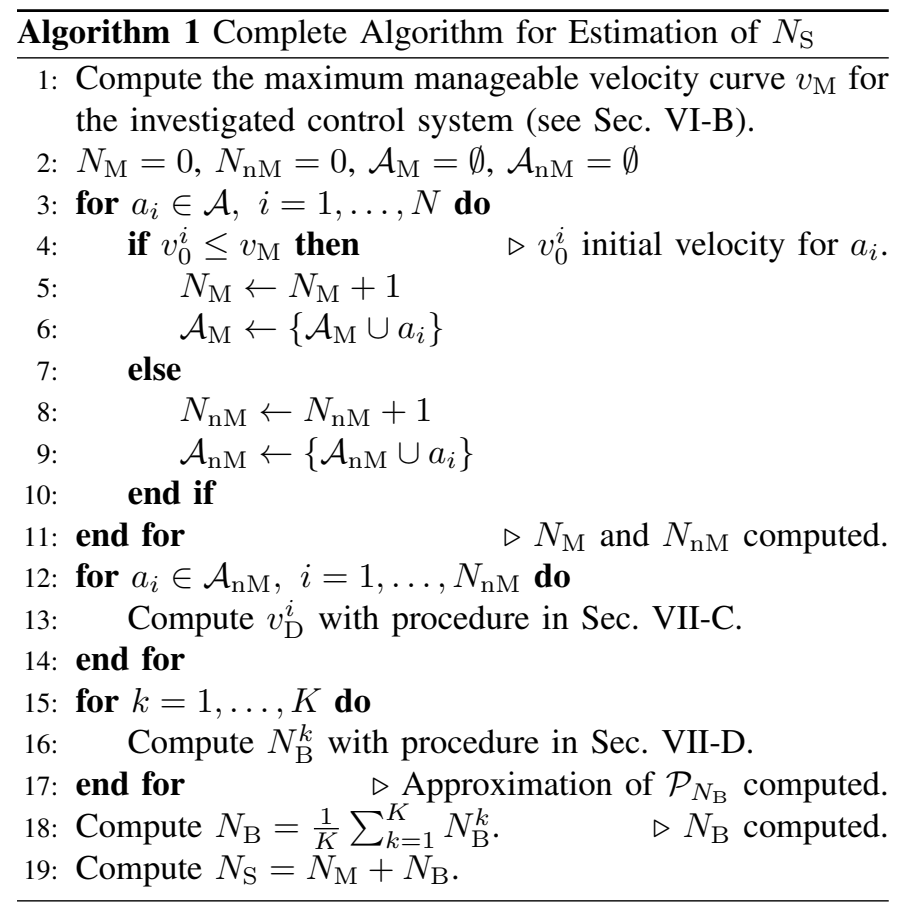

reduced effect of impact is summarized in a formal algorithm. Subsequently, the results from application of the method on the road-departure accidents from GIDAS are presented.

\section{A. Complete Algorithm}

The developed approaches for computation of maximum manageable velocity curves, prediction of departure velocity, and estimation of mitigation presented in Sec. VI-B, Sec. VII-C, and Sec. VII-D, respectively, comprise the complete algorithm for estimating the potential of the new safety system in terms of avoided accidents or reduction of severity in an unavoidable accident. The complete computational procedure is given in Algorithm 1. The total number $N_{\mathrm{S}}$ of accidents from the database that resulted in severe injuries or fatalities, which potentially could be avoided based on the analysis of the new autonomous control system is then obtained by addition of the number $N_{\mathrm{M}}$ of manageable accidents and the number $N_{\text {B }}$ of mitigated accidents according to

$$
N_{\mathrm{S}}=N_{\mathrm{M}}+N_{\mathrm{B}}
$$

This complete algorithm is general in the sense that it can handle any scenario that can be formulated using the cost function and constraints (5)-(10), including parameter variations. Examples of other scenarios described in this form can be found in, e.g., [16], [17].

\section{B. Results for the Road-Departure Accidents in GIDAS}

The quantitative results obtained by applying Algorithm 1 on the road-departure accidents in Fig. 2 are presented in this section. The number of severe road-departure accidents filtered out from GIDAS is 233, and thus $N=233$. The number of manageable accidents, i.e., those accidents below the maximum manageable velocity curve $v_{\mathrm{M}}$ in Fig. 5 is 177 for $\mathrm{AYC}$ and 190 for O-LKC, and thus $N_{\mathrm{M}}=177$ for
TABLE II

COLLECTION OF RESULTS OBTAINED BY ANALYZING THE ROAD-DEPARTURE ACCIDENTS IN GIDAS (WITH FILTERING ACCORDING TO TABLE I) USING THE METHOD IN ALGORITHM 1.

\begin{tabular}{|c|c|c|c|c|c|}
\hline & $\boldsymbol{N}$ & $\boldsymbol{N}_{\mathbf{M}}$ & $\boldsymbol{N}_{\mathbf{B}}$ & $\boldsymbol{N}_{\mathbf{S}}$ & $\boldsymbol{N}_{\mathbf{S}} / \boldsymbol{N}(\%)$ \\
\hline AYC & 233 & 177 & 6 & 183 & 79 \\
\hline O-LKC & 233 & 190 & 7 & 197 & 85 \\
\hline
\end{tabular}

AYC and $N_{\mathrm{M}}=190$ for O-LKC as obtained in Sec. VI-C. It is also the result in Step 11 in Algorithm 1 for the respective control principle, AYC and O-LKC. Recall that all these accidents could potentially be avoided by the use of the autonomous control principle according to the analysis, which means that the associated severe or fatal injuries are also avoided. In addition to these accidents predicted to be manageable, there are the possible mitigations obtained by the fact that these control systems decrease the velocity by braking, and thus mitigate the collision in the cases where the entry velocity was non-manageable. These results were presented in Sec. VII-E and are the outcome at Step 18 in Algorithm 1. For AYC, the number of non-manageable accidents are $N_{\mathrm{nM}}=N-N_{\mathrm{M}}=233-177=56$, and of these mitigation by braking could potentially avoid $N_{\mathrm{B}}=6$. For O-LKC, the number of non-manageable accidents are $N_{\mathrm{nM}}=N-N_{\mathrm{M}}=233-190=43$, and of these mitigation by braking could potentially avoid $N_{\mathrm{B}}=7$. The following total predicted number of accidents with reduced degree of injury or avoided fatalities are thus obtained

$$
\begin{aligned}
\text { AYC: } & N_{\mathrm{S}}=N_{\mathrm{M}}+N_{\mathrm{B}}=177+6=183, \\
\text { O-LKC: } & N_{\mathrm{S}}=N_{\mathrm{M}}+N_{\mathrm{B}}=190+7=197 .
\end{aligned}
$$

The resulting numbers for the control principles AYC and OLKC are collected in Table II.

1) Value of Autonomy: The resulting numbers for the roaddeparture accidents collected in Table II can now, in relation to the total number $N=233$ of severe road-departure accidents extracted from GIDAS, be used for comparisons. As described in Sec. IV, the control principle AYC can be considered in two ways, either as an autonomous system of lower complexity than O-LKC or as an AYC system with an alert driver that reacts as good as can be hoped for in the critical situation. A first approach to draw conclusions is to consider that autonomous lane-keeping control with O-LKC could potentially avoid $N_{\mathrm{S}} / N=85 \%$ of the severe accidents and the corresponding number for AYC is $N_{\mathrm{S}} / N=79 \%$. The overall conclusion is consequently that autonomy has a very high potential in future safety systems, considering that even an autonomous control system of lower complexity potentially could avoid $79 \%$ of the road-departure accidents with severe injuries or fatalities.

2) Comparing two Autonomous Control Strategies: A second type of conclusion to draw from the obtained results is interesting as a guidance to investigate the potential effects of developing a system of lower complexity or a comparably more complex autonomous safety system. In the example considered here, the O-LKC principle requires an optimal steering solution for lane keeping whereas for AYC it suf- 
fices to implement the straightforward steering law (1). It is plausible that the development costs (and also the required hardware costs in each vehicle) for O-LKC are larger than the corresponding costs for AYC. This should be weighted against the fact that $\mathrm{O}-\mathrm{LKC}$ is $(197-183) / 183=7.7 \%$ better in performance according to the analysis. There is a large number of severe road-departure accidents world-wide, and in that perspective $7.7 \%$ represent many mitigated severe injuries or even avoided fatalities.

3) Comparing O-LKC to AYC with a Human Driver: A third type of analysis is to compare a potential new system, here O-LKC, with an existing system, here AYC. Such a comparison highly depends on the assumptions on the driver. If the driver is highly alert, reacts immediately and correctly, then the maximum velocity curve $v_{\mathrm{M}}$ for $\mathrm{AYC}$ would be the same as for the idealized AYC considered in the analysis here, and the result in terms of improvement obtainable with O-LKC, $7.7 \%$, would also be the same. Any other assumption on the driver would be straightforward to include as follows. Assume that the car is driven autonomously and suddenly a critical situation appears, where the driver is expected to retake control of the vehicle. It then has to be assumed that a certain reaction time is required for the driver, and in addition a spectrum of possible behaviors, including the steering law (1), but also more critical like an over-reaction. Then a new maximum manageable velocity curve should be computed based on the actual driver model (DM). The new maximum manageable velocity curve $v_{\mathrm{M}}^{\mathrm{DM}}$ and the predicted departure velocities $v_{\mathrm{D}}$ are computed with respect to delays and other expected behaviors, and Algorithm 1 can then be used in the same way as in the presented analysis.

\section{Discussion-Velocity Reduction}

It is of interest to study in what velocity intervals, reduction of velocity could significantly decrease the risk of a severe or fatal injury. To this purpose, the injury risk function shown in Fig. 6 is considered. For low or very high velocities, the possible effect of the control system is weak, since the risk function is comparably flat in these regions. In between, approximately in the interval $30-70 \mathrm{~km} / \mathrm{h}$, the derivative of the risk function with respect to velocity is large and velocity reduction could have a significant effect on the risk of an accident. Therefore, O-LKC provides an additional benefit in that it reduces the velocity more before an inevitable impact than AYC. For the road-departure accidents in $\mathcal{A}_{\mathrm{nM}}$ in the analysis, the average velocity reduction for AYC is $4.4 \%$ and for O-LKC the corresponding number is $13.7 \%$.

\section{Discussion-Policy and Strategy Aspects}

Referring to the research question raised in Sec. I regarding complexity of systems for vehicle autonomy, it is of interest to investigate what conclusions can be drawn when it comes to potentially reduced number of severe or fatal accidents, and what that could mean in terms of future policies and strategies for autonomy development. Out of the 233 road-departure accidents in the filtered selection from the database GIDAS, the optimal autonomous strategy O-LKC could potentially avoid 197 severe accidents, which is 14 more than AYC with a correctly acting driver, also interpreted as autonomous AYC with a steering controller as in (1). The latter control strategy could consequently potentially avoid 183 accidents with severe injuries or fatalities. This result means that the vast majority of potentially avoided severe or fatal accidents, 183, is a result of vehicle autonomy, and that optimal autonomous steering and braking could increase this number with 14 additional severe accidents to 197 . Thus, an autonomous strategy of comparably lower complexity could avoid a majority (183), whereas a much more complex system could avoid even more (197). If the study would have shown that the autonomous safety system of lower complexity could avoid few severe or fatal accidents but the more complex system could avoid significantly more, then the research and development for autonomy should strive for realization of the more complex ones already in the short perspective. Instead, the overall conclusion is that autonomy by itself makes the most significant change for increasing traffic safety and that there is a high potential also in a system with lower complexity. This is an encouraging result for continuing the step-wise introduction of new autonomy features in passenger vehicles towards the goal of full autonomy.

\section{CONCLUSIONS}

A method has been developed to predict the potential value of future autonomous vehicle maneuvers at-the-limit of tire friction in safety-critical situations, and lane departure has been used as the example scenario. A key component is how data from crash databases with actual previous accidents are utilized in the algorithm to predict the value of future, not yet existing systems. Another enabling fact has been the developments in readily available tools for numerical optimization, which now makes it possible to perform optimization of different variations of parameters in accident scenarios. For the tool used here, JModelica.org, the development in performance and usability where computations now take seconds or minutes compared to hours some years ago, is also a key enabler. This has the consequence that it is tractable to compute the maximum manageable velocity $v_{\mathrm{M}}$ and predict the departure velocity $v_{\mathrm{D}}$ several times in the algorithm (given the parameters for the specific scenario from the database). It is also a contribution in the paper that the computation of the departure velocity can be performed with the same optimization method by using the strategy of successively increasing the lane width $d$. Thus, the method handles analysis of both manageable accidents and mitigation of non-manageable accidents when complete avoidance is predicted not to be possible. Overall, the presented approach works effectively and makes it possible to use large data sets from real crash databases together with numerical optimization of vehicle behavior.

The method was used on a set of 233 road-departure accidents, with fatal or severe injuries, from the GIDAS crash database. Two control strategies, AYC and O-LKC, were evaluated, and they were selected so that one, AYC, represents a straightforward extension of ESC with a steering law simply based on road curvature whereas the second, O-LKC, represents an advanced system based on optimal steering in 
combination with optimal braking strategies. The main result is that an autonomous lane-keeping system in critical situations could potentially avoid the vast majority of severe accidents, here $79 \%$ and $85 \%$ for AYC and O-LKC, respectively. This effect is achieved by either managing to keep the vehicle in the driving lane, or by reducing the velocity before an impact such that the resulting injuries are not severe or fatal. From a perspective of strategies for future development, the final overall conclusion is that autonomy in itself is a key enabler and therefore, when it comes to reducing the number of severe or fatal accidents, it is important to strive for the realization of autonomous vehicle-safety systems.

\section{ACKNOWLEDGMENTS}

The authors would like to acknowledge Dr. Nils Lubbe at Autoliv AB for generous support with data extraction from the GIDAS database and subsequent interpretation of the data, as well as Prof. Mattias Villani at the Div. of Statistics and Machine Learning at Linköping University for interesting discussions regarding the statistical approach.

\section{REFERENCES}

[1] Bundesanstalt für Straßenwesen (BASt), "Unfallforschung - GIDAS," Accessed: 2018-11-07. [Online]. Available: https://www.bast.de/BASt_ 2017/DE/Fahrzeugtechnik/Fachthemen/f2_gidas.html

[2] The Swedish Transport Agency, "STRADA-Informationssystem för olyckor och skador i trafiken," Accessed: 2018-11-07. [Online]. Available: https://www.transportstyrelsen.se/en/road/statistik/STRADA/

[3] S. A. Ferguson, "The effectiveness of Electronic Stability Control in reducing real-world crashes: A literature review," Traffic Injury Prevention, vol. 8, no. 4, pp. 329-338, 2007.

[4] S. Sternlund, J. Strandroth, M. Rizzi, A. Lie, and C. Tingvall, "The effectiveness of lane departure warning systems-A reduction in realworld passenger car injury crashes," Traffic Injury Prevention, vol. 18, no. 2, pp. 225-229, 2017.

[5] T. Gordon et al., "Advanced crash avoidance technologies (ACAT) program-Final report of the Volvo-Ford-UMTRI project: Safety impact methodology for lane departure warning-Method development and estimation of benefits," U.S. Dept. Transportation, National Highway Traffic Safety Administration, Tech. Rep., 2010, No. DOT HS 811405.

[6] C. Erbsmehl, "Simulation of real crashes as a method for estimating the potential benefits of advanced safety technologies," in Proc. $21 s t$ Int. Tech. Conf. Enhanced Safety of Vehicles (ESV2009), no. 09-0162, Stuttgart, Germany, 2009.

[7] K. Kusano, T. I. Gorman, R. Sherony, and H. C. Gabler, "Potential occupant injury reduction in the US vehicle fleet for lane departure warning-equipped vehicles in single-vehicle crashes," Traffic Injury Prevention, vol. 15, no. sup1, pp. S157-S164, 2014.

[8] K. D. Kusano and H. C. Gabler, "Comparison of expected crash and injury reduction from production forward collision and lane departure warning systems," Traffic Injury Prevention, vol. 16, no. sup2, pp. S109S114, 2015.

[9] S. Tanaka, T. Mochida, M. Aga, and J. Tajima, "Benefit estimation of a lane departure warning system using ASSTREET," SAE Int. J. Passenger Cars-Electronic and Electrical Systems, vol. 5, no. 1, pp. 133-145, 2012.

[10] E. Y. M. Teraoka, S. Tanaka, and T. Mochida, "Benefit estimation method for lane departure warning systems in the american traffic environment," SAE Technical Paper, Tech. Rep., 2014, No. 2014-010172 .

[11] H. Stigson, A. Kullgren, and E. Rosén, "Injury risk functions in frontal impacts using data from crash pulse recorders," in Proc. 56th AAAM Annual Conf., Ann Adv Automot Med., vol. 56, 2012, pp. 267-276.

[12] International Organization for Standardization (ISO), "ISO 262621:2018 Road vehicles - Functional safety," 2018.

[13] U. Kiencke and L. Nielsen, Automotive Control Systems, For Engine, Driveline, and Vehicle, 2nd ed. Berlin Heidelberg, Germany: Springer Verlag, 2005.
[14] K. Lundahl, B. Olofsson, K. Berntorp, J. Åslund, and L. Nielsen, "Towards lane-keeping electronic stability control for road-vehicles," in Proc. 19th IFAC World Congress, Cape Town, South Africa, 2014, pp. 6319-6325.

[15] M. Klomp, M. Lidberg, and T. J. Gordon, "On optimal recovery from terminal understeer," Proc. Institution of Mechanical Engineers, Part D: J. Automobile Engineering, vol. 228, no. 4, pp. 412-425, 2014.

[16] K. Berntorp, B. Olofsson, K. Lundahl, and L. Nielsen, "Models and methodology for optimal trajectory generation in safety-critical roadvehicle manoeuvres," Vehicle System Dynamics, vol. 52, no. 10, 2014.

[17] V. Fors, B. Olofsson, and L. Nielsen, "Formulation and interpretation of optimal braking and steering patterns towards autonomous safety-critical maneuvers," Vehicle System Dynamics, vol. 57, no. 8, pp. 1206-1223, 2019.

[18] J. Åkesson, K.-E. Årzén, M. Gäfvert, T. Bergdahl, and H. Tummescheit, "Modeling and optimization with Optimica and JModelica.orgLanguages and tools for solving large-scale dynamic optimization problems," Computers and Chemical Engineering, vol. 34, no. 11, pp. 17371749, Nov. 2010

[19] N. Lubbe and M. Ranovona, "Simulation of lane departure accidents in Germany," in Proc. 3rd Int. Symp. Future Active Safety Technology Towards zero traffic accidents (FAST-zero), Gothenburg, Sweden, 2015, pp. $115-122$.

[20] H. B. Pacejka, Tyre and Vehicle Dynamics, 2nd ed. Oxford, United Kingdom: Butterworth-Heinemann, 2006.

[21] S. E. Mattsson and H. Elmqvist, "Modelica-An international effort to design the next generation modeling language," IFAC Proceedings Volumes, vol. 30, no. 4, pp. 151-155, 1997, 7th IFAC Symp. Computer Aided Control Systems Design (CACSD), Gent, Belgium.

[22] A. T. van Zanten, "Bosch ESP systems: 5 years of experience," SAE Technical Paper, Tech. Rep., 2000, no. 2000-01-1633.

[23] R. Yu, M. Quddus, X. Wang, and K. Yang, "Impact of data aggregation approaches on the relationships between operating speed and traffic safety," Accident Analysis \& Prevention, vol. 120, pp. 304-310, 2018.

[24] P. Choudhary, M. Imprialou, N. R. Velaga, and A. Choudhary, "Impacts of speed variations on freeway crashes by severity and vehicle type," Accident Analysis \& Prevention, vol. 121, pp. 213-222, 2018.

[25] D. R. Cox, "The regression analysis of binary sequences," J. Royal Statistical Society: Series B (Methodological), vol. 20, no. 2, pp. 215232, 1958

[26] T. A. Gennarelli and E. Wodzin, "AIS 2005: A contemporary injury scale," Injury, vol. 37, no. 12, pp. 1083-1091, 2006, Special Issue: Trauma Outcomes.

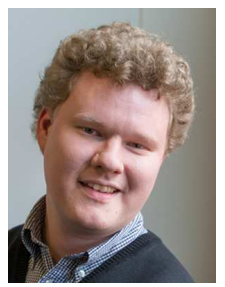

Björn Olofsson received the M.Sc. degree in Engineering Physics in 2010 and the Ph.D. degree in Automatic Control in 2015, both from Lund University, Sweden. He is currently a researcher at the Division of Vehicular Systems, Linköping University and at the Department of Automatic Control, Lund University. His research interests are in motion control for robots and vehicles, optimal control, system identification, and statistical sensor fusion.

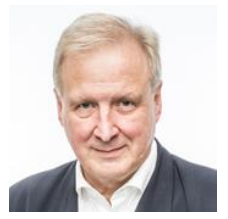

Lars Nielsen holds the Sten Gustafsson Chair in Vehicular Systems at Linköping University, Sweden. He received his M.Sc. in Engineering Physics in 1979 and his Ph.D. in Automatic Control in 1985, both from Lund University, Sweden. His main research interests are automotive modeling, control, and autonomy. 